\title{
FIBER OPTIC SENSORS FOR ENVIRONMENTAL MONITORING
}

\author{
Leonid B. Likumovich, \\ Andrei V. Medvedev, \\ Oleg I. Kotov, \\ Sergei I. Markov, \\ Vladimir M. Nikolaev \\ St.Petersburg State Technical University, Russia,
}

\begin{abstract}
Sensors discussed are designed to register acoustic waves and micro deformations in ground. Sensor technology is based on phase modulation that occurs in optical fiber when it is influenced by outside mechanical disturbance. In our paper we consider two possible ways of registration this phase modulation: fiber optic interferometer and mode to mode interference. These methods can be incorporated with various signal processing technique to obtain maximum efficiency of fiber optic sensor under the certain conditions.
\end{abstract}

It is well known that optical fibers are widely used to transmit high band signals for long distances. In this case fiber sensitivity to environmental disturbances is a bad thing. But from other side the disturbances change propagating light properties (intensity, phase, polarization, etc.). This changes can be registered in the output light and after appropriate signal processing will give information about the parameters of the outside influence on the fiber. This is an idea in brief how optical fibers can be used as physical parameters sensors. 
As an example a simple sensor that consists of the laser source, optical fiber, photo detector and signal processing unit can be discussed (Fig. 1). The main idea of the system is to register micro disturbances of the fiber

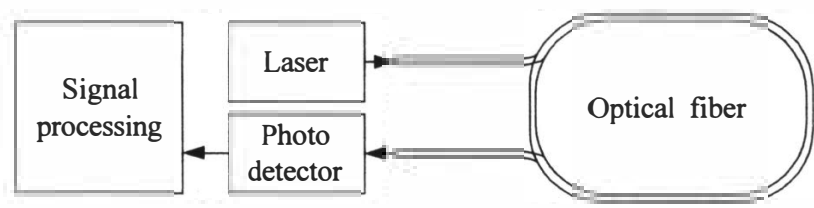

Fig.1 A simple fiber optic sensor that may be caused by the outside mechanical influences on it. The micro strains that occur under this conditions results in phase modulation of the light propagating in the optical fiber. This phase modulation can be recovered by means of the fiber interferometer or using modemode interference.

The first method gives extra high sensitivity to the system - optical fiber length variations up to $10^{-9}$ meter can be registered. But it needs coherent laser source and single mode polarization maintaining optical fiber. Using of other types of fibers makes the magnitude of the interferometer signal not constant, depending on slow temperature variations and fading sometimes up to zero levels.

The advantages of using the mode-mode interference method are: using of multi mode optical fiber reduces the requirements to other optical elements, mode-mode interference signal is not so sensitive - so it is easier to suppress signal produced by temperature fluctuations of the fiber. Sensitive fiber up to several kilometers long can be used.

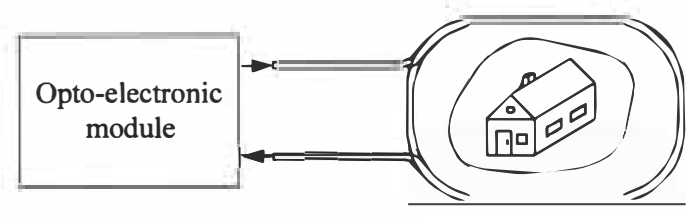

Fig.2 Fiber optic security sensor

The system with multi mode fiber was used as a security device (Fig.2). Phase modulation was caused by an unwanted visitor walking on the earth surface where the sensitive fiber was buried in (Fig.3). The signal was registered and processed in opto-electronic module. We obtained the sensitivity of the system

enough to register the person walking on the ground just over the fiber or jumping in 10-15 meters from it. This parameter was limited not by sensor characteristics but by the additional noises existing in the ground (from transport, for example). Several systems of this type with sensor element from 50 to 800 meters long were incorporated into security systems to indicate the unwanted penetration to the protected area.

Fiber optic sensing element of the interferometer type was used in sound sensor (Fig.4). We used high-coherent semiconductor laser, optical isolator and unbalanced Mach-Zhender fiber interferometer. For signal

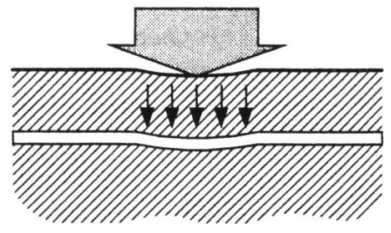

Fig.3 
processing we used additional phase modulation produced by laser source current scanning. So we designed remote sensor with passive full fiber sensing element and fiber connecting link. Maximum sensitivity of the sensor with 50 meter long sensitive fiber was $10^{-4}-10^{-5} \mathrm{~Pa} / \mathrm{Hz}^{1 / 2}$ in $300-10000 \mathrm{~Hz}$ frequency range.

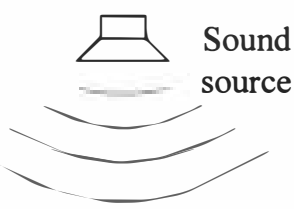

The sensor can be used not only for air acoustic waves measuring but also in the ground sounds detecting. In both cases two sensor configurations are possible: with concentrate (Fig.5,a) and distributed (Fig.5, b) sensor element. The second one may be more interesting

Fig.4 Fiber optic microphone comparing to traditional (not fiber) sensor systems because it uses fiber property to integrate the outside influence along its length. Using of several sensors with appropriate signal processing let us construct sensor array and get complex data about acoustic wave field in the ground.

We also used interferometer type sensing element in fiber optic temperature probe (Fig.6).

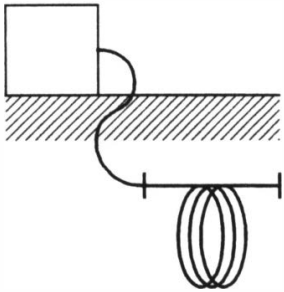

a) concentrated

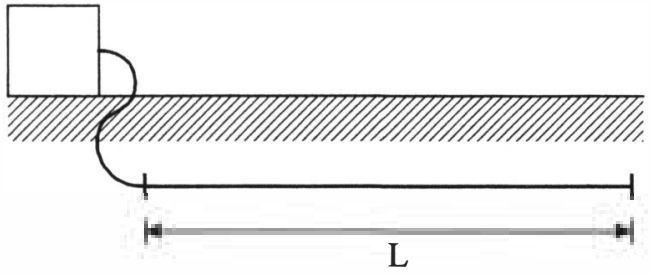

b) distributed

Fig.5 Sensor element configurations

With 10 meter fiber Fabry-Perot interferometer we had sensitivity about $10^{-3}$ degree in -10 $+100^{\circ} \mathrm{C}$ temperature range using very simple signal processing method. 


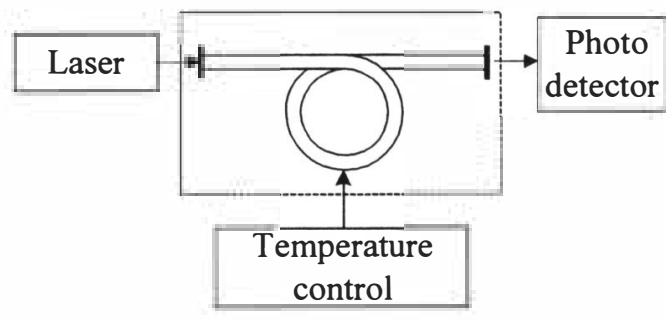

Fig.6 Fiber optic temperature sensor
In temperature sensor also concentrate and distributed sensor elements can be used. Interferometers can be incorporated in consecutive sensor system (Fig.7) or one interferometer can be used to scan temperature field. The last method together with tomographic methods and computer signal processing was used to investigate temperature distributions over heating object. The result of the reconstructing of the temperature field over the C-shaped heated wire is

depicted in Fig.8.

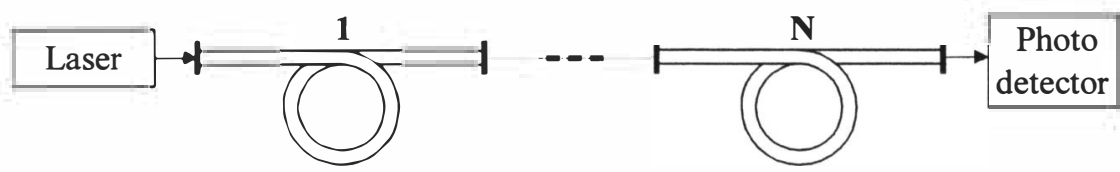

Fig.7 Consecutive multi element interferometer sensor system.

Complex analysis and real time processing of characteristics measured by fiber optic sensors together with data obtained by traditional types of sensors can give information that may be important in environmental monitoring, earth shakes prediction, etc.

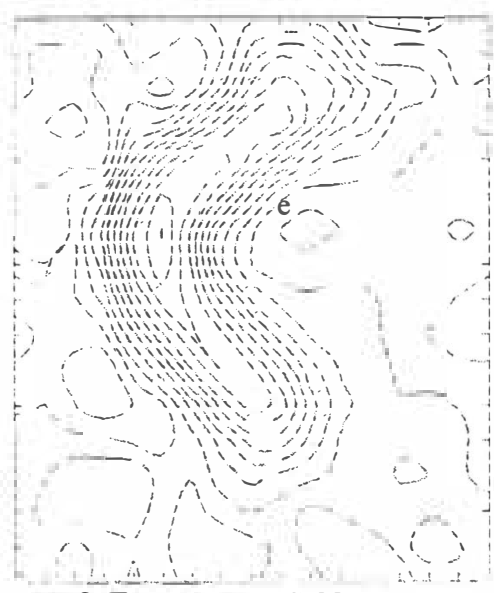

Fig.8. Temperature field topogram 